\title{
THE INTERNAL/EXTERNAL QUESTION
}

\author{
Philip HUGLY \& Charles SAYWARD \\ University of Nebraska/Lincoln
}

\section{Introduction}

Rudolph Carnap expresses his famous distinction between the internal question and external question thus:

What is now the nature of the philosophical question concerning the existence or reality of numbers? To begin with, there is the internal question which, together with the affirmative answer can be formulated ... by "There are numbers" ... This statement follows from the analytic statement "five is a number" and is therefore itself analytic. Moreover it is rather trivial ... therefore nobody who meant the question "Are there numbers?" in the internal sense would either assert or seriously consider a negative answer. This makes it plausible to assume that those philosophers who treat the question of the existence of numbers as a serious philosophical problem and offer lengthy arguments on either side, do not have in mind the internal question ... Unfortunately, these philosophers have so far not given a formulation of their question in terms of the common scientific language. Therefore our judgement must be that they have not in giving the external question and to the possible answers any cognitive content. ${ }^{\text {' }}$

As a question internal to arithmetic, the question 'Are there numbers?' has a trivial affirmative answer. As a question external to arithmetic it has no cognitive content.

A fundamental question in the philosophy of mathematics is whether there are numbers. Realists produce 'There are numbers'; anti-realists produce 'There aren't any numbers'. These philoso-

1. Rudolph Carnap, "Empiricism, Semantics and Ontology". Reprinted from Revue Internationale de Philosophie, 4 (1950), in Irving M. Copi and James A. Gould (editors), Contemporary Philosophical Logic (New York, 1978), p. 153. 
phers take themselves to be thereby making assertions on which they differ - one asserting what the other denies. For Carnap it is not the case that there is either assertion or denial in such exchanges, although, doubtlessly, it feels like assertion and denial.

This essay defends Carnap's view of the matter. We differ on details but agree on the bottom line that either 'There are numbers' has a content unsuitable for a philosophy of mathematics or it has no content at all.

\section{A realist objection}

A realist objection to Carnap goes like this: Carnap admits that 'There are numbers' is a true sentence of arithmetic. So it is true that there are numbers. This is the fundamental thesis of realism. So what Carnap says hardly is an objection to realism. Now it is true that Carnap also says that what 'There are numbers' says within arithmetic differs from what it says when asserted by a mathematical realist. But this is just obscure - too obscure to bother with. Carnap says that ' 5 is a number' is analytic, and, since 'There are numbers' follows from it, Carnap holds that 'There are numbers' is also analytic. So Carnap might have responded to part of this realist objection by saying that, since 'There are numbers' is analytic, it is not suitable for a metaphysical claim. This is not a persuasive reply to the objection. The thesis that the theorems of arithmetic are analytic is implausible, and it is not a point on which we would wish to defend Carnap.

\section{3. 'Number' within mathematics}

Both Carnap and his realist opponent take it as obvious that 'There are numbers' is a sentence of arithmetic. But this is not obvious. After all 'There are numbers' contains no mathematical signs. In developing what we call natural number theory we use the signs for addition, multiplication, equality and the numerals, along with such letters as ' $n$ ' and ' $m$ ' together with signs for generality and sentential composition. 
Among these signs is not to be found a sign corresponding to the word 'number' in the kind of grammatical application it is given in 'There are numbers'.

The mathematical sign 'number' is the word together with its use in numerical quantifiers (as in 'For every number $\mathrm{n}$ there is some number $m$ such that $m$ is greater than $\left.n^{\prime}\right)$. That sign is absent from 'There are numbers'. And, apart from its use in numerical quantifiers, the word plays no role in arithmetic.

We need to distinguish between the primitive vocabulary of arithmetic and the defined vocabulary. The primitive vocabulary is very sparse, consisting of the signs for addition, multiplication, equality, the numerals, the signs for generality and sentential composition. (It can be rendered even more sparse with the numerals giving way to two signs: one for zero and one for the successor function.)

But, in addition, there are signs which are defined in terms of the primitive vocabulary. For example, to say $n$ is even is to say that, for some $\mathrm{m}, \mathrm{n}=2 \times \mathrm{m}$.

So there is the option of introducing into arithmetic a predicative use of 'number'. All that is required is that we select some numerical formula Fn which is correct no matter what numeral we put for ' $n$ '; for example,

for some $m, m=n+1$

will serve as a definition of

$\mathrm{n}$ is a number

thus conferring a predicative use of 'number' within arithmetic.

But if ' $\mathrm{n}$ is a number' is to be taken as short for 'for some $\mathrm{m}$, $m=n+1^{\prime}$ then the philosopher's 'There are numbers' comes to nothing other than

For some $n$ and $m, m=n+1$

in its mathematical sense.

Now ask yourself whether the content of mathematical realism the signs for ng with such nd sentential

$s$ that "There not obvious. cor admits that ers' is also inalytic, it is uasive reply ithmetic are would wish 
is entirely mathematical in nature so that the propositions of mathe matical realism are nothing other than the theorems of arithmetic.

This picture of a person who from time to time - and perhaps in books and articles - writes down various well-known arithmetical quantifications and calls it a philosophy of mathematics fits nothing with which we are familiar.

Someone says 'There are no numbers'. The Zen master replies' with a theorem of arithmetic, and perhaps lays out its proof. $\mathrm{He}$ meets every such challenge in just this way. He might also, from time to time, apply the conclusicns of certain proofs in some practical way. One might call him a philosopher, but one would not say that what he says constitutes a philosophy of mathematics.

In any case, the mathematical realist is no Zen master. The realist wants to add something - namely, that these theorems and proofs are not about nothing - that there are things (that there are things), which they are about - numbers.

But to add this is to utter a sentence in addition to those of mathematics, and so to utter a sentence not a part of mathematics, and thus not a sentence the sense of which is secured by its place within mathematics.

Realists may present various proofs within mathematics and make various assertions within mathematics. But then they want to add something, and try to do so with such words as 'There are numbers'. This is something they can't so much as try to do if they stick with the sentences of mathematics. So, we cannot show that the realist's sentence makes sense by noting that it is just another mathematical sentence for which it is unproblematic that it makes sense.

(And what holds for the realist holds as well for the antirealist who disputes realism by negation.)

If 'number' is short for some mathematical formula, then 'There are numbers' is just another quite ordinary mathematical sentence, one of a kind which goes virtually undisputed. And if 'number' is not short for some mathematical formula, then one certainly cannot show that 'There are rumbers' has a sense by showing that it is a sentence of mathematics!

4. Model the

A realist obje assertion 'Th is an assertio del theory sh

$\exists$ n $n=0$

is true only if ' $n$ ' ranges $-\mathrm{i}$ are numbers.

Modal the idea is to defi then to define

The functi variable $v$ of second, $\mathrm{f}($ ' 0 ' any terms $t a$

Truth is $t 1$ $\left(\mathrm{t}=\mathrm{r}^{2}\right)$ is tru formulas $\mathrm{A}$ : only if $\mathrm{A}$ is 1 only if $\mathrm{A}$ is 1 ' $\exists \mathrm{VA}^{\prime}$ ' is true except at mc Within th

$\exists \mathrm{n} \mathrm{n}=0$ $\mathrm{n}, \mathrm{n}=0$

are derivabl theory it is 1

But, som 'exists', bul 'some'.

The wor 'there exist: 
ositions of mathems of arithmetic. - and perhaps in lown arithmetical natics fits nothing

en master replies out its proof. $\mathrm{He}$ might also, from proofs in some ut one would not mathematics. naster. The realist rems and proofs there are things)

ition to those of of mathematics, ured by its place

nathematics and hen they want to ds as 'There are try to do if they cannot show that it is just another tic that it makes

or the antirealist ula, then 'There natical sentence, id if 'number' is certainly cannot wing that it is a

\section{Model theory}

A realist objection to all of this goes as follows: Even if the realist assertion 'There are numbers' is not a sentence of mathematics, it is an assertion that is justified by thinking about mathematics. Model theory shows us that a simple arithmetic quantification such as

$\exists \mathrm{n} \quad \mathrm{n}=0$

is true only if the class of numbers - the class over which the variable ' $n$ ' ranges - is non-empty, and that class is non-empty only if there are numbers.

Modal theory for arithmetic is done in different ways. The key idea is to define a function on the terms of the object language, and then to define truth relative to this function.

The function, call it ' $\mathrm{f}$ ', satisfies these conditions: First, for any variable $v$ of the object language, $f(v)=n$, for some number $n$; second, $f\left({ }^{\prime} 0^{\prime}\right)=0$; third, for any term $t, f\left({ }^{(} t^{\prime}\right)=f(t)+1$; fourth, for any terms $t$ and $r, f\left(t^{(}+r^{\prime}\right)=f(t)+f(r)$, and $f\left({ }^{\prime}\left(x r^{\prime}\right)=f(t) x f(r)\right.$

Truth is then defined relative to $f$. First, for any terms $t$ and $r$, $\left(t=r^{2}\right)$ is true relative to $f$ if and only if $f(t)=f(r)$. Second, for any formulas $A$ and $B$, and variable $V, \sim A$ ' is true relative to $f$ if and only if $A$ is not true relative to $f$; 'AvB' is true relative to $f$ if and only if $A$ is true relative to $f$ or $B$ is true relative to $f$; and, finally, ' $\exists v A$ ' is true relative to $f$ if and only if, for some function $g$ like $f$ except at most that $f(v) \neq g(v)$,A is true relative to $g$.

Within this framework such familiar biconditionals as

' $\exists \mathrm{n} \mathbf{n}=0$ ' is true relative to $\mathrm{f}$ if, and only if, if for some number $\mathrm{n}, \mathrm{n}=0$

are derivable. What has been set out is a model theory and in this theory it is nowhere said that numbers exist.

But, someone might object, we have managed to avoid the word 'exists', but not the concept of existence - for we used the word 'some'.

The word 'some' is used. But we could just as well have used 'there exists'. Nothing turns on using one phrase or another. No one 
thinks that arithmetic changes if we everywhere use 'some' instead of 'exists' or vice versa.

It makes no difference whether we use ' $\exists$ n', 'for some n', 'for some number n' or 'there exists a number $n$ such that'. Unless there is a predicative use of 'number' in the language of the model theory - that is to say, ordinary mathematical English - we cannot infer 'There exists a number' from 'There exists a number $\mathrm{n}$ such that ... n ...'.

An examination of the model theory for arithmetic set out above reveals no sign corresponding to the word 'number' in the kind of grammatical application it is given in "There are numbers" or in 'There exists a number'. The sign 'number' is used in numerical quantifiers. But apart from this use the sign plays no role in model theory.

\section{5. 'Number' outside mathematics}

Perhaps it is of no significance that we can do mathematics without the predicate 'number'. Perhaps we actually use the word predictively outside of mathematics.

That appears to be how we use it when, for example, we distinguish between colors and numbers. We say that three is a number, but that red isn't.

Or just imagine the use of mathematics in a physical theory. We there can use 'number' in a predicative manner so as to distinguish, e.g., particles from numbers.

It is like the case with sets. Pure set theory does without any sign for sets - but only because in pure set theory our domain consists of sets alone. But the domains of those languages in which set theory finds an application are not thus limited-and within them a predicative sign for sets finds a use.

So we now need to consider such sentences as 'Colors are not numbers', 'Red is not a number', 'Particles aren't numbers', and 'Tables aren't sets':

Suppose that the following is a truth formulable in the language of some theory of color including at least elementary arithmetic:

For every $x$, if $x$ is $c$

We will not want to inf

If red is odd, then re

and to bar this inferenc instead

For every $\mathrm{x}$, if $\mathrm{x}$ is a

But if we do, we can th

If red is a number th

which is equally unwa

For every number $\mathrm{x}$

which yields

If 3 is odd, then 3 is

but not

If red is odd, then re

So what is needed to

'number', but the use o for letters used to expr is the practice of replar And so once again we $\dot{c}$ a distinction among thi The fundamental poi for color and number dc word won't in general the use of number word of 3 and 2' nor ' 3 is brig 
me' instead

ome n', 'for Unless there odel theory cannot infer such that.

et out above the kind of abers' or in i numerical le in model

tics without ord predic, we distina number, theory. We distinguish, ut any sign in consists h set theory a predica-

ors are not nbers', and e language ithmetic:
For every $\mathrm{x}$, if $\mathrm{x}$ is odd, $\mathrm{x}$ is not even

We will not want to infer, e.g.,

If red is odd, then red is not even

and to bar this inference we could use the word 'number' and write instead

For every $\mathrm{x}$, if $\mathrm{x}$ is a number then if $\mathrm{x}$ is odd, $\mathrm{x}$ is not even.

But if we do, we can then infer

If red is a number then if red is odd, then red is not even

which is equally unwanted. What we really need is

For every number $\mathrm{x}$, if $\mathrm{x}$ is odd, then $\mathrm{x}$ is not even

which yields

If 3 is odd, then 3 is not even

but not

If red is odd, then red is not even.

So what is needed to guard against nonsense is not a predicate 'number', but the use of that word to delimit acceptable substitutes for letters used to express generalities. And what is essential here is the practice of replacing certain letters only by numerical terms. And so once again we do not need the word as a predicate - to draw a distinction among things.

The fundamental point would seem to be that the language games for color and number don' $t$ 'intersect'. A term with the use of a color word won't in general yield a sense when it replaces a term with the use of number word, and conversely. Neither 'Red is the product of 3 and 2' nor ' 3 is brighter than pink' make sense. We can of course 
count each not true, and in that sense false. But in that sense a cat says anything. In that sense opener also is false.

Just as 'number' serves as an index to generalization, and thus i Objection 2. You need to re dispensable, so also for 'color'. Suppose we lacked this word. Wit may help if you look at the yet might say that Joseph had a coat of many colors by using foncept of number, and so sure sentence like

inder it.

Reply. There is no doubt th

For many $f$, Joseph's coat was $f$

where it was our practice to recognize as instances of this general Not every concept is a co ization only such sentences as

Joseph's coat was red

Joseph's coat was blue

But what kind of concept is it

here are concepts of that kind

xistence (as expressed in a st

concept of that kind, as is st

his concept is a sign for gener erm (e.g., a noun).

It may well be that the cor and the like - that is, as we would say, to recognize as instances oarithmetical language. But $w$. this generalization only sentences formed with words for colors concept of number? Might it $r$. words with that kind of use.

\section{Further objections and replies}

We construct formulas using by numerals and carry out cert from these by replacing letter

Won't it be (roughly) our $\xi$ grasp of the concept number

Objection 1. This whole involuted enquiry is predicated on thethathematical predicate. (Anc supposition that there is a doubt whether 'There are numbers' makehumbers' is the kind of conce sense. But there is no plausibility in this so-called doubt. The word Remember: Even if we l at issue are plain English. You may claim not to understand the language of natural number tr sentence 'There are numbers', but you understand it nonethelessactual use of 'number' is for This doubt is just a pretense, and the enquiry to which it has led hads like that of a subscript wh: no point.

Reply. The sentence 'There are numbers' has a normal grammal and its words are familiar ones. Does that show that it has a sense If it did, it would show as well that the sentence 'Three is red' has' a sense. And it isn't obvious that it has a sense.

We grant that it may be that everybody does perfectly welt understand this sentence, that it does make sense and that everybody grasps the sense it makes. It may be that it is only a false philosophy which keeps us from seeing clearly that this sentence makes a sense to go in for the letters.

Objection 3. You asked fo that 'There are numbers' mak something which supports th sense? And if you couldn't, that it does? we grasp. But - for whatever reason - it yet is not clear to us that tion using 'beet', and then as 
that sense a calt says anything. In that sense our doubt is a real one, even if it is founded on nothing but the obscurities in our own thought.

tion, and thus Objection 2. You need to relax and learn to accept the obvious. 1 this word. Wt may help if you look at the matter this way: We all grasp the lors by using foncept of number, and so surely can conceive that something falls inder it.

Reply. There is no doubt that we grasp the concept of number. But what kind of concept is it? Is it a concept of a kind such that here are concepts of that kind under which things fall?

of this general Not every concept is a concept of that kind. The concept of xistence (as expressed in a sentence like 'There are lions') is not concept of that kind, as is shown by the fact that what expresses his concept is a sign for generality (e.g., a quantifier), not a general erm (e.g., a noun).

It may well be that the concept of number is expressed by our as instances ofrithmetical language. But what about that language expresses the ds for colors foncept of number? Might it not be the letters ' $m$ ', ' $\mathrm{n}$ ' and the like? We construct formulas using these letters and replace these letters by numerals and carry out certain inferences with formulas resulting from these by replacing letters by numerals.

Won't it be (roughly) our grasp of all this which constitutes our grasp of the concept number - not our mastery of one or another icated on themathematical predicate. (And what we would need for 'There are mbers' make humbers' is the kind of concept expressed by a predicate.)

bt. The word Remember: Even if we had 'number' as a predicate in the nderstand thelanguage of natural number theory, it would be entirely useless. Our nonetheless actual use of 'number' is for the expression of generality. It's use it has led has s like that of a subscript which reminds us which expressions are to go in for the letters.

mal grammal Objection 3. You asked for something which supports the claim thas a sense that 'There are numbers' makes sense. But why? Could you produce ee is red' has something which supports the claim that 'There are beets' makes sense? And if you couldn't, would that at all sap your confidence erfectly wel that it does?

at everybody That we are not sure how to show that a sentence makes sense is e philosophyno reason whatsoever for doubting that it does make sense.

nakes a sense Reply. Consider the case of beets. Someone might read a descripear to us thation using 'beet', and then ask to be shown that there are such roots. 
We know how to respond to this request. We bring various roots an see whether any fits the description, and find one does. We the agree: Yes, there are beets.

There is something analogous for 'square of 27 '. An easy calcu lation shows that 729 is a square of 27 . Having carried it out, w will then agree that, yes, there are squares of 27.

But the case for number doesn't fit this familiar pattern. Who description do we have for 'number', so that we can decide whethe 27 fits that description? Shall we say that a number is a timeles entity? By what method might we find out that 27 fits that descrip tion? Or, what calculation shows that 27 is a number? No calculatiol shows any such thing.

A calculation shows e.g., that 27 is $13+4+10$. That 27 is number is not something which can be brought out within mathe matics.

Someone unfamiliar with our notation might ask whether $27 \mathrm{i}$ a number. We could then exhibit our use of that sign. They would then be satisfied. ' 27 is a number' can be used to express a recogni tion about the use of a sign.

Objection 4. This is just so much palaver. The key point gets los in all this talk. It is clear that we all realize that three is a numbe and that red, for example, isn't. And this recognition can easily b expressed in words - as easily as it has just been expressed in words!

Reply. If there is a recognition here, then it might be lacking. So let us suppose that someone failed to recognize that three is number. What would they have missed? And how might their failure be remedied?

We here imagine a person who counts, adds, multiplies, applies the results of adding and multiplying, etc. We imagine a persor reasonably competent in the empirical application of mathematica terms, and in the arithmetic of those terms. This is enough to have him be one who grasps the idea of three. But he is supposed to fail to recoqnize that three is a number.

So now we tell him something about numbers. Our hope is that once he gets this information he'll recognize that three is a number

What do we tell him? Shall we say that a number is a timeless, placeless entity?
The doubt whether anythin s matched by the doubt whet timeless' and the like.

\section{Final remarks}

Both Carnap and his (imagina 'number' within mathematics. numbers' is a theorem of arit arithmetic is actually set out $r$ no predicative use within ari between odds and evens, for $e$ And how these distinctions ge up predicate like 'number'.

It is true that one could co infinitely many degree-one for Fn

which become true sentences ' $n$ '. The formula

$$
\mathrm{n}=\mathrm{n}
$$

is an example. If 'number' is we say, with Carnap, that it is im of mathematical realism com sentences as ' $\exists n n=n$ ' or tha down to the denial of such sen Carnap was on target with hi numbers from a point of view $\mathrm{e}$ numbers such as that five is on don't exist are unmathematica A final point Carnap made unmathematical claims lack $c$ matics, 'number' has no clear matics; rather, it is a sign for $\mathrm{g}$ 
us roots an es. We the easy calct d it out, $w$

ittern. Wh

ide whethe a timeles hat descrip calculatio

hat 27 is thin math lether 27 hey woul a recogn

nt gets los s a numbe $\mathrm{n}$ easily b pressed acking. S three is neir failur es, applie a persoi thematica gh to hav sed to fai

pe is tha a number timeless
The doubt whether anything is asserted by 'There are numbers' is matched by the doubt whether anything is asserted by 'Three is timeless' and the like.

\section{Final remarks}

Both Carnap and his (imaginary) realist critic had it wrong about 'number' within mathematics. They each thought that 'There are numbers' is a theorem of arithmetic. But an examination of how arithmetic is actually set out reveals no such thing. "Number' has no predicative use within arithmetic. Distinctions such as those between odds and evens, for example, get made in familiar ways. And how these distinctions get made does not draw on any cooked up predicate like 'number'.

It is true that one could cook up such a predicate. There are infinitely many degree-one formulas

Fn

which become true sentences no matter which numeral is put for ' $n$ '. The formula

$$
\mathrm{n}=\mathrm{n}
$$

is an example. If 'number' is defined by some such formula, then we say, with Carnap, that it is implausible to suppose that the content of mathematical realism comes down to the assertion of such sentences as ' $\exists \mathrm{n} n=\mathrm{n}$ ' or that the content of anti-realism comes down to the denial of such sentences.

Carnap was on target with his point that philosophers speak about numbers from a point of view external to mathematics. Claims about numbers such as that five is one of them, that they exist, or that they don't exist are unmathematical claims.

A final point Carnap made with which we agree is that these unmathematical claims lack content. As is the case inside mathematics, 'number' has no clear use as a predicate outside of mathematics; rather, it is a sign for generality. 